\title{
Effect of Inspiratory Maneuvers on Lung Deposition of Tobramycin Inhalation Powder: A Modeling Study
}

\author{
Jennifer J. Meerburg, MD, ${ }^{1,2}$ Eleni-Rosalina Andrinopoulou, PhD, ${ }^{3}$ Aukje C. Bos, MD, PhD, ${ }^{1,2}$ \\ Hwain Shin, $\mathrm{PhD},{ }^{4}$ Marcel van Straten, $\mathrm{PhD},{ }^{2}$ Kamal Hamed, MD, ${ }^{5}$ \\ Paul Mastoridis, PharmD, ${ }^{5}$ and Harm A.W.M. Tiddens, MD, PhD ${ }^{1,2}$
}

\begin{abstract}
Background: Tobramycin inhalation powder (TIP) and tobramycin inhalation solution (TIS) are considered equally effective for the treatment of chronic pulmonary Pseudomonas aeruginosa infection in cystic fibrosis (CF) patients. The impact of TIP inhalation maneuvers on distribution of tobramycin is unknown. We hypothesized that (1) fast TIP inhalations result in greater extrathoracic and reduced small airway concentrations compared with slow or uninstructed TIP inhalations; (2) slow TIP inhalations result in greater small airway concentrations than TIS inhalations. The aim of the study was to assess TIP and TIS deposition with computational fluid dynamics (CFD).

Methods: Uninstructed, instructed fast, and instructed slow TIP inhalations of CF patients on maintenance TIP therapy, and inhalations during nebulization of saline with PARI LC Plus ${ }^{\circledR}$ were recorded at home. Drug deposition was determined using TIP and TIS aerosol characteristics together with CFD simulations based on airway geometries from chest computed tomography scans. The drug concentration was assessed in extrathoracic, central, large, and small airways.

Results: Twelve patients aged 12-45 years were included, and 144 CFD simulations were performed. In all individual analyses, the tobramycin concentrations were well above the threshold for effective dose of 10 times minimal inhibitory concentration throughout the bronchial tree. Extrathoracic concentrations were comparable between fast and uninstructed TIP inhalations, while slow inhalations resulted in reduced extrathoracic concentrations compared with uninstructed TIP inhalations $(p=0.024)$. Small airway concentrations were comparable between fast and uninstructed TIP inhalations, while slow TIP inhalations resulted in greater small airway concentrations than uninstructed TIP inhalations $(p<0.001)$. Small airway concentrations of TIS were comparable with those of slow TIP inhalations $(p=0.065)$, but greater than those of fast and uninstructed TIP inhalations $(p<0.001)$.
\end{abstract}

Conclusion: All TIS and TIP inhalation maneuvers resulted in high enough concentrations, however, inhaling TIS or inhaling TIP slowly results in the greatest small airway deposition.

Keywords: cystic fibrosis, dry powder inhalers, inhalation therapy, nebulizers, pulmonary medicine, tobramycin

\footnotetext{
${ }^{1}$ Department of Pediatric Pulmonology and Allergology, Erasmus Medical Center, Sophia Children's Hospital, Rotterdam, the Netherlands.

Departments of ${ }^{2}$ Radiology and Nuclear Medicine and ${ }^{3}$ Biostatistics, Erasmus Medical Center, Rotterdam, the Netherlands.

${ }^{4}$ Novartis Pharma AG, Basel, Switzerland.

${ }^{5}$ Novartis Pharmaceuticals Corporation, East Hanover, New Jersey.
} 


\section{Introduction}

$\mathbf{C}$ YSTIC FIBROSIS (CF) is a serious hereditary disease. The airways of patients with $\mathrm{CF}$ are covered by a layer of viscous mucus, resulting in impaired mucociliary clearance of pathogens. CF patients are particularly prone to chronic pulmonary infection by Pseudomonas aeruginosa $(\mathrm{Pa})$, which induces chronic inflammation leading to progressive structural damage of the lung. Damaged lungs are a good environment for $\mathrm{Pa}$ to survive, and when not eradicated $\mathrm{Pa}$ can transform to a mucoid form, which is more resistant to treatment. $^{(1)}$ With increasing age, more patients become chronically infected with $\mathrm{Pa}$. According to annual registries from $2016,31 \%$ of patients with CF in Europe and $29 \%$ of patients in the United States are chronically infected with $P a{ }^{(2,3)}$ Fortunately, these chronic infections can be suppressed effectively with maintenance inhaled antibiotic therapy. Longterm inhaled antibiotic therapy is recommended in this case by both the European and U.S. CF guidelines. ${ }^{(4,5)}$

For chronic $\mathrm{Pa}$ infections, nebulization therapy with tobramycin inhalation solution (TIS) has been widely studied. ${ }^{(6-8)}$ However, this therapy is relatively time-consuming, nebulizers require thorough cleaning after use, and technical maintenance. Therefore, tobramycin inhalation powder (TIP), a formulation using spray-dried porous particles (PulmoSphere ${ }^{\mathrm{TM}}$ ) administered with a T-326 inhaler (Podhaler ${ }^{\mathrm{TM}}$ ), was developed as a more patient-friendly therapy. The T-326 inhaler is pocket-size, requires minimal cleaning, is disposable after 1 week of use, and administration takes only 5 minutes. ${ }^{(9)}$

Noninferiority studies showed similar efficacy (forced expiratory volume in 1 second and $P a$ colony forming units in the sputum) and pharmacokinetic characteristics of TIP compared with TIS. ${ }^{(9,10)}$ However, in the phase III EAGER trial $(n=553)$, sputum concentrations of TIP were almost twice as high as those of TIS. ${ }^{(10)}$ This could possibly be explained by high deposition of tobramycin in the extrathoracic and large airways. Whether this results in a difference in tobramycin concentration in the small airways between TIP and TIS is unknown.

It is important to get the antibiotic to the area where $P a$ is located. $P a$ is ubiquitous in the lungs, and found in both large and small airways, and even in the alveoli. ${ }^{(11)}$ Due to major morphological changes in CF patients starting early in life, efficient targeting of the small airways is important. ${ }^{(12,13)}$ The concentrations of inhaled antibiotics in the small airways need to be well above the minimal inhibitory concentration (MIC) for effective killing of $\mathrm{Pa}{ }^{(1)}$ Consequently, it is believed that treatment efficacy of inhaled antibiotics might be improved by maximizing deposition of the antibiotic in the small airways.

To date, it is not known whether the concentrations of tobramycin in the small airways after inhalation of TIP and TIS are above the MIC, as this is extremely hard to measure in vivo. To estimate concentrations of inhaled antibiotics throughout the bronchial tree, computational fluid dynamic (CFD) models have been developed. ${ }^{(14)}$ These in silico models allow us to improve our insight on the relationship between aerosol particle characteristics, inhalation maneuvers, disease severity, and small airway antibiotic concentrations. Using these CFD models, it was shown that for aztreonam lysine for inhalation, high inspiratory flows during nebulization resulted in a suboptimal treatment compared with low inspiratory flows. ${ }^{(1)}$ To our knowledge, small airway concentrations of TIP and TIS have not been studied using CFD models. There are reasons to expect differences in deposition patterns in the small airways between both formulations. The particle characteristics of the TIP and TIS formulations are different, and the inhalation of TIP versus TIS requires different inhalation maneuvers. TIS is inhaled with the use of a PARI LC Plus ${ }^{\circledR}$ (PARI Medical Holding GmbH, Starnberg, Germany) nebulizer and Porta$\mathrm{Neb}^{\circledR}$ compressor (Philips Respironics, Chichester, United Kingdom) while the patient is breathing quietly in and out with low flow rates until the complete dose is nebulized, which can take up to 20 minutes. ${ }^{(15)}$ On the contrary, TIP is inhaled through specific breathing maneuvers consisting of a full expiration followed by deep inspiration. It takes one or two of these maneuvers to empty one capsule of TIP, and one dose comprises four capsules. As patients are instructed to inhale at least twice per capsule, up to eight inhalations are needed to inhale one dose of TIP. Of note, the T-326 inhaler for TIP has a low to medium airflow resistance $(0.08$ square root of a centimeter $\left.\mathrm{H}_{2} \mathrm{O} * \mathrm{~L} / \mathrm{min}\right){ }^{(16)}$ As a result, patients inhaling TIP show a wide variation of inspiratory flow rates between 40 and $120 \mathrm{~L} / \mathrm{min}^{(17)}$ In vitro experiments showed that the total lung dose of TIP is relatively unaffected by differences in flow rate. (17) However, the deposition pattern of this dose in the large and small airways is unknown.

Based on the above, we formulated the following research questions: (1) Is tobramycin deposition for TIP in the large and small airways influenced by inspiratory flow rate? (2) Are deposition patterns of TIP different from that of TIS, and do they both result in concentrations of tobramycin that are high enough throughout the lungs?

The aim of our study was to assess the deposition patterns of TIP and TIS using CFD simulations based on threedimensional (3D) airway models of computed tomography (CT) scans of patients with $\mathrm{CF}$, for various patient-specific flow rates.

We hypothesized that high inspiratory flow rates of TIP would result in greater extrathoracic and reduced small airway tobramycin concentrations compared with low flow rates. In addition, we hypothesized that for TIP inhaled with low inspiratory flow rates, small airway concentrations would be higher relative to TIS inhalations with tidal volume breathing.

\section{Materials and Methods}

\section{Study design}

This study is an in silico study based on CFD simulations with use of flow profiles from an observational study.

\section{Patient selection}

For the computational investigation, we used the recorded inspiratory flows of the first 12 patients who were enrolled in a larger observational study of TIP and TIS (NTR5212). Inclusion criteria for the observational study were a diagnosis of $\mathrm{CF}$, age 6 years and older, and maintenance treatment with TIP for at least 1 month. Exclusion criteria were pulmonary exacerbation defined as the need for intravenous 
antibiotics at the time of the inspiratory flow registrations, and inability to follow instructions. Both adolescents and adults from four Dutch CF centers were included: Amsterdam University Medical Center, Erasmus Medical Center (Rotterdam), Haga Teaching Hospital (The Hague), and University Medical Center Utrecht.

\section{Flow recordings}

The inspiratory flows were recorded during two study visits that took place at the patient's home using an inhalation profile recorder (The Technology Partnership, Cambridge, United Kingdom) as described before by Haynes et al. ${ }^{(17)}$ In short, the inhalation profile recorder was adjusted to the T-326 inhaler or the PARI LC plus nebulizer with an extra mouthpiece that fitted closely. This mouthpiece was connected with a tube to a pressure gauge, which was connected to a transducer and a laptop. With this system, the pressure drop over the mouthpiece during inhalation was measured and plotted as flow/time curve (area under the curve representing the inhaled volume). The flow was calculated by dividing the square root of pressure drop by resistance. For the T-326 inhaler, the inhaler resistance was 0.088 square root of a centimeter $\mathrm{H}_{2} \mathrm{O} * \mathrm{~L} / \mathrm{min}$. For the PARI LC Plus, the inhaler resistance was 0.030 square root of a centimeter $\mathrm{H}_{2} \mathrm{O} * \mathrm{~L} / \mathrm{min}$.

For TIP simulations, the inspiratory flows during inhalation of tobramycin with the T-326 inhaler were recorded. Three inhalation maneuvers for each patient were measured during two study visits: an uninstructed, an instructed fast, and an instructed slow inhalation. At the first study visit, the uninstructed inhalation was measured after patients were asked to inhale TIP in the way they would normally do. At the second study visit, the instructed fast and slow inhalation were measured as follows: patients were asked (in random order) to inhale one TIP capsule as fast as possible, and to inhale another TIP capsule as slow as possible, but with a flow that was high enough to let the capsule rattle in the T326 inhaler.

For the TIS simulations, inspiratory flows were recorded while inhaling $0.9 \%$ saline with a Porta-neb compressor and PARI LC Plus nebulizer during the second home visit. The inspiratory flows during nebulization were measured in four recordings, of 15 seconds each. From these four recordings, a mean inhalation curve was computed. Expiratory flows were not recorded, but computed, using the recorded inhaled volume and total time between inhalations.

\section{CFD modeling of aerosol deposition}

CFD modeling was used to simulate aerosol deposition in $3 \mathrm{D}$ computer lung models. This method has been extensively described elsewhere. ${ }^{(14,18)}$ In short, the flow dynamics within lung models are determined to predict the course and velocity of the particles after they are virtually injected into the model. To assess the internal flow distribution, the differences between lobar volumes on expiratory and on inspiratory CT scans were used. With this technique, local airway deposition of inhalation medication can be computed in patient-specific models. The CFD model has been validated against single-photon emission computed tomography (SPECT$\mathrm{CT}),{ }^{(18)}$ and has been used in $\mathrm{CF}^{(19)}$ and non-CF (asthma/ chronic obstructive pulmonary disease) studies. ${ }^{(14,20-22)}$ CFD model simulations were performed in Fluent 14.0 (Ansys, Inc., Canonsburg, PA).

\section{$3 D$ model reconstruction}

To execute CFD modeling, nine 3D lung models were reconstructed using chest $\mathrm{CT}$ scans from patients with $\mathrm{CF}$. An image of a reconstructed 3D model showing a coupled mouthpiece, the extrathoracic, central, and large airways is shown in Supplementary Data S1. The process is extensively described elsewhere. ${ }^{(19)}$ Briefly, the following five steps were carried out:

First, we selected nine CT scans of a data set from Sophia Children's Hospital ( $n=187$ patients) of routinely acquired biennial chest CT scans of patients with CF. The selection criteria of the CT scans are described in the next paragraph.

Second, the central and large airways were reconstructed from the chest CT scans. The central airways are defined as the area from the trachea up to the lobar bronchi. The large airways, often referred to in the literature as the distal airways, are defined as the airways from the first segmental bronchi to airways with a diameter of $\pm 1-2 \mathrm{~mm}$ that are still visible on chest CT scan.

Third, the extrathoracic airways, defined as the mouth and throat, including the larynx, until the trachea, were reconstructed. These airways were not imaged on the chest CT scans of the patients. The minimal cross-sectional diameter is decisive for extrathoracic airway deposition. ${ }^{(23)}$ Therefore, an adult extrathoracic airway model with a median minimal cross-sectional diameter was selected from a database $(n=105)$ of FLUIDDA NV (Kontich, Belgium). The model was then scaled down such that both the anteroposterior and lateral dimension of the scaled model's trachea at the location of the sternum matched the lung model.

Fourth, CAD models of the T-326 inhaler or PARI LC plus mouthpiece were connected to the mouth of the model.

Fifth, the small airways were defined for each lung lobe. Small airways, also referred to in literature as peripheral airways, are airways with a diameter smaller than $\pm 1-2 \mathrm{~mm}$. These airways are not visible on chest CT images and therefore cannot be reconstructed from a CT scan. Instead, their surfaces were determined using Phalen's description of the airway tree in infants, children, and adolescents, based on the patient's height. ${ }^{(24)}$

\section{Chest CT selection}

The selected CT scans had to meet the following technical requirements: volumetric, spirometer-controlled; availability of both inspiratory and expiratory scans; and a maximum slice thickness of $1 \mathrm{~mm}$ or smaller. Furthermore, CT scans had to match the TIP-TIS study population as closely as possible based on age, height, and gender. For this purpose, we divided our study patients into three subpopulations: adolescents and female and male adults. We calculated the average height of each subpopulation. The height of the patients for whom we used CT scans differed by a maximum of $5 \%$ from the average height of the corresponding subpopulation.

The CT scans from the Sophia database were obtained from patients younger than 18 years. For the female adult and male adult subpopulations, CT scans from adolescents 
after they were fully grown were selected, as determined by a flattened individual growth curve.

In addition to age, CT scans of patients with different disease severities were selected. Therefore, three lung models were reconstructed per subpopulation, each with a different lung disease severity category: mild, moderate, and severe.

To assess the severity of CF lung disease on CT scan, we used the validated Perth-Rotterdam Annotated Grid Morphometric Analysis for cystic fibrosis (PRAGMA-CF) scoring method, ${ }^{(25)}$ which is a morphometric method using a grid to score CT images for percentage of lung disease. Using this score, the extent of bronchiectasis, mucus plugging, and airway wall thickening was computed. The differentiation between mild, moderate, and severe disease was based on the combination of these subscores. To select CT scans within each severity category, we first divided the scans in the Sophia database according to our three subpopulations (adolescents and female and male adults). Next, all CT scans were ranked according to the outcomes of the PRAGMA-CF scores for percentage disease. We defined the lowest tertile as mild, the middle tertile as moderate, and the upper tertile as severe disease. Finally, one CT scan within each tertile was selected for each subpopulation, resulting in the selection of nine CT scans. In addition, the selected CT scans were reviewed unblinded by a pediatric pulmonologist (H.A.W.M.T.) to evaluate whether in his opinion, the disease severity corresponded to the selected severity group. This evaluation confirmed that the selection represented a good spread of disease severity for each of the three subpopulations.

An overview showing how the flow profiles from the three subpopulations were applied on the CT models is presented in Figure 1.

\section{Aerosol characteristics}

For TIP, particle characteristics are dependent on flow rate. In general, when the flow rate through the T-326 inhaler increases, the mass median aerodynamic diameter (MMAD) of the generated particles decreases. With a nextgeneration impactor (NGI), particle characteristics, including the MMAD, geometric standard deviation, fine particle fraction, and delivered dose, were derived for three different inspiratory flows $(40,60,85 \mathrm{~L} / \mathrm{min})$ (Table 1).

The mean flow rate of each simulated inhalation was calculated, and the particle characteristics (aerodynamic particle size distribution) of the flow that was closest to this mean flow were selected. A single inhalation maneuver was simulated and the deposited mass scaled to the total of four capsule inhalations. We assumed that the complete capsule dose of TIP would be released after an inhaled volume of $1.2 \mathrm{~L}$ (data on file). Although it is required to inhale TIP twice, it was shown that most patients are able to empty the capsule in a single inhalation. ${ }^{(26)}$ To strengthen our statistical models (all patients having the same amount of inhalations), only the first inhalation was simulated. We did not check after each inhalation whether the capsule was emptied but instead relied on the inhaled volume. If a patient was not able to inhale with a volume of $1.2 \mathrm{~L}$, the dose of TIP injected into the model was adjusted relatively to the inhaled fraction of $1.2 \mathrm{~L}$. In the TIP simulations, we did not account for exhalations as the patient instructions list a breath holding of 5 seconds, during which the medication is assumed to be deposited. ${ }^{(27)}$

For TIS, we considered the characteristics of nebulized droplets to be flow independent. We used aerosol characteristics (MMAD, geometric standard deviation, fine particle fraction, and delivered dose) that were measured with an NGI at a flow rate of $15 \mathrm{~L} / \mathrm{min}$ for all CFD computations (Table 2). In each individual TIS simulation, TIS was continuously released until a full dose of $5 \mathrm{~mL}(300 \mathrm{mg}$ of tobramycin) would have been nebulized: the injected dose was calculated by doubling the deposited dose from the NGI, as the NGI based on a 1:1 inhalation-exhalation cycle and no particles can enter the system during exhalation. Next, the patient's specific inhalation-exhalation cycle was stimulated. Exhaled drug (drug that left the model during expiration phase) and drug released by the nebulizer during expiration were considered lost to the environment. Thus, the exhalation time of an individual patient had a large impact on the total amount of lost drug.

For both TIP and TIS, we accounted for dead volume by using the delivered doses of the NGI as the starting point for injected dose, as the dead volume is not measured with the NGI and thus excluded by default.
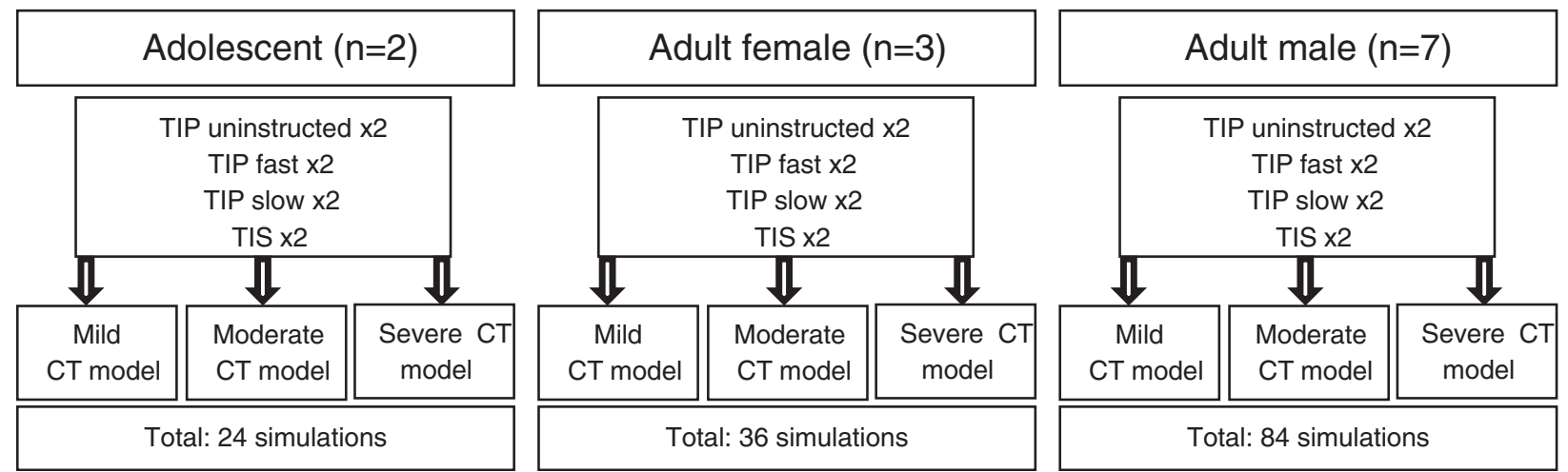

FIG. 1. Overview of simulations. An overview of how the flows of the three groups (adolescent, adult female, and adult male) were applied on the CT models is presented in this figure. For every patient, we collected an uninstructed, instructed fast, and instructed slow inhalation of TIP, and we calculated a mean inhalation of TIS from 60 seconds of recording. Every flow was used as input on three different CT models. This resulted in a total of 144 simulations with computational fluid dynamics modeling. CT, computed tomography; TIP, tobramycin inhalation powder; TIS, tobramycin inhalation solution. 
Table 1. Particle Characteristics Assessed WITH NEXT-GENERATION IMPACTOR

\begin{tabular}{lccccr}
\hline Treatment & $\begin{array}{c}\text { Flow } \\
(\text { L/min })\end{array}$ & $\begin{array}{c}\text { MMAD } \\
(\mu m)\end{array}$ & $\begin{array}{c}\text { GSD } \\
(\mu \mathrm{m})\end{array}$ & $\begin{array}{r}\text { FPF } \\
(\%)\end{array}$ & $\begin{array}{r}\text { DD } \\
(\%)\end{array}$ \\
\hline TIP & 40 & 3.8 & 1.7 & 57 & 95 \\
TIP & 60 & 3.3 & 1.7 & 59 & 102 \\
TIP & 85 & 3.0 & 1.8 & 61 & 103 \\
TIS & 15 & 4.2 & 2.4 & 58 & 35 \\
\hline
\end{tabular}

Particle characteristics of TIP and TIS derived from tests with next-generation impactor. The impactor was not cooled. These data were used as starting point for fluid dynamic modeling simulations.

DD, delivered dose; FPF, fine particle fraction; GSD, geometric standard deviation; MMAD, mass median aerodynamic diameter; TIP, tobramycin inhalation powder; TIS, tobramycin inhalation solution.

\section{Tobramycin concentration}

To compute tobramycin concentrations throughout the bronchial tree, the surface area of each reconstructed airway and of all the combined small airways was calculated. We only accounted for deposition in the 3D models, as we assumed that tobramycin would not be deposited in the alveoli. Concentrations of tobramycin in $\mu \mathrm{g} / \mathrm{mL}$ were computed for the extrathoracic and central airways and for each lung lobe, with distinction between the large and small airways. To assess the deposition with CFD, we made the following assumptions. First, we assumed that particles were deposited once they touched the airway walls. Second, we assumed that particles delivered beyond the reconstructed airway model were distributed evenly over the small airway surface area (determined by the Phalen model as described above). Third, we assumed the thickness of the airway surface liquid to be constant to compute concentrations. The ratio of deposited drug mass and airway surface liquid volume of each lung region is defined as its averaged drug concentration. These concentrations were computed for three different scenarios: a moderate thin $(3 \mu \mathrm{m}),{ }^{(28)}$ in between $(5 \mu \mathrm{m})$, and a thick layer $(7 \mu \mathrm{m})$ of airway surface liquid. ${ }^{(29)}$ For the concentrations of deposited drug presented in this article, the airway surface liquid thickness was $7 \mu \mathrm{m}$ (worst-case scenario). Concentrations for the other lining fluid layer scenarios can easily be calculated by dividing the outcomes by $7 \mu \mathrm{m}$ and multiplying them by 3 or $5 \mu \mathrm{m}$.

\section{Effective tobramycin concentration}

To set the tobramycin concentration for effective inhibition of $P a$ growth, we selected as starting point an MIC of $4 \mu \mathrm{g} / \mathrm{mL}$, as described by the European Committee on Antimicrobial Susceptibility Testing (EUCAST) (www.eucast .org/clinical_breakpoints/). However, we defined an effective conservative tobramycin concentration as 10 times MIC, that is, $40 \mu \mathrm{g} / \mathrm{mL}$, as there are many factors such as mucus binding and mucociliary clearance that may negatively affect the activity of tobramycin. ${ }^{(1)}$

\section{Statistical analysis}

Patient characteristics are tabulated using descriptive statistics, categorical data being presented as counts $(n)$ and proportions $(\%)$. Continuous data are presented as median and interquartile ranges (IQRs). The outcomes of the assessment are shown in box-and-whisker plots. To investigate differences in concentrations between different TIP inhalation maneuvers and differences in concentration between TIP and TIS, generalized estimating equation models for correlated data were used. These models account for multiple measurements within each patient. The reference conditions for the models were uninstructed TIP inhalations to assess differences within TIP, and TIS inhalations to assess differences between TIP and TIS. CT scan model and lung region were considered possible confounders. To check whether our results were biased by patients who did not receive a full dose of TIP because not all their inhaled volumes reached the needed $1.2 \mathrm{~L}$ to empty a capsule, sensitivity analyses were done without those patients. Results of testing are considered significant if the $p$-value is below 0.05 . SPSS/PC statistics (SPSS, Inc. Chicago, IL) and statistical software package R, version 3.4.3 (free download from www.rproject .org) were used for the statistical calculations.

\section{Ethics}

Written informed consent for the use of deidentified data was obtained from the patients and/or their parents or guardians depending on their age. Approval for both the observational study and the Sophia database was obtained by the Institutional Review Board of Erasmus Medical Center, Rotterdam, the Netherlands (MEC-2015-329 and MEC-2013-078, respectively).

\section{Results}

\section{Patient selection}

The patient population selected from the clinical study consisted of 4 females (33\%) and 8 males (66\%). Patients were divided into the three subpopulations as follows: adolescent $(n=2)$, adult female $(n=3)$, and adult male $(n=7)$. Patient demographics and lung function data are presented in Table 2.

Table 2. Patient Characteristics

\begin{tabular}{lccc}
\hline Patient & Sex & Age (years) & FEV $_{1} \%$ predicted $^{\mathrm{a}}$ \\
\hline 1 & $\mathrm{M}$ & 12 & 104 \\
2 & $\mathrm{~F}$ & 13 & 94 \\
3 & $\mathrm{M}$ & 18 & 92 \\
4 & $\mathrm{M}$ & 20 & 63 \\
5 & $\mathrm{M}$ & 22 & 72 \\
6 & $\mathrm{M}$ & 25 & 106 \\
7 & $\mathrm{M}$ & 25 & 110 \\
8 & $\mathrm{M}$ & 25 & 49 \\
9 & $\mathrm{~F}$ & 29 & 35 \\
10 & $\mathrm{M}$ & 35 & 60 \\
11 & $\mathrm{~F}$ & 41 & 80 \\
12 & $\mathrm{~F}$ & 45 & 95 \\
\hline
\end{tabular}

${ }^{\mathrm{a}}$ Best $\mathrm{FEV}_{1} \%$ of the year before inclusion, using global lung initiative 2012 references. Patients were divided into three groups based on sex and age: adolescents (patients 1 and 2), adult females (patients 9, 11, and 12), and adult males (patients 3, 4, 5, 6, 7, 8, and 10).

$\mathrm{F}$, female; $\mathrm{FEV}_{1}$, forced expiratory volume in 1 second; $\mathrm{M}$, male. 


\section{Flow recordings}

The patients inhaled TIP with a wide range of flows. Figure $2 \mathrm{a}-\mathrm{c}$ shows the inspiratory flow curves of the uninstructed, instructed fast, and instructed slow TIP inhalations. All patients performed an instructed fast inhalation that was faster than the uninstructed inhalation. Similarly, the instructed slow inhalation for all patients was slower than the uninstructed inhalation. Except for two situations, all patients were able to inhale more than the minimum volume of 1.2 $\mathrm{L}$ needed to inhale the full content of the capsule. One patient inhaled only $0.5 \mathrm{~L}$ through the $\mathrm{T}-326$ inhaler with the instructed fast inhalation, and another patient inhaled only $0.7 \mathrm{~L}$ with the uninstructed inhalation. For the modeling of this maneuver, the inhaled dose of TIP was adjusted to $42 \%$ and $58 \%$, respectively.

For TIS, mean inspiratory flow curves from the same population that were calculated for each individual ranged from 0.2 to $2.4 \mathrm{~L}$.

\section{Tobramycin concentration}

Figure 3 illustrates the computed tobramycin concentrations throughout the bronchial tree for uninstructed, in- structed fast, and instructed slow inhalations of TIP and the TIS inhalations for three single-study subjects and for three of the nine CT models.

In Figure 4, the box-and-whisker plots summarized the computed tobramycin concentrations in the large and small airways of all simulations for each flow type and device. Large airway concentrations were up to 100 -fold greater compared with small airway concentrations, which is a result of the much smaller airway surface in the large airways compared with the small airways. Median (IQR) concentration in the large airways was 73,597 (41,587-126,353) $\mu \mathrm{g} / \mathrm{mL}$, which is 1839 times larger than the threshold value. Median (IQR) concentration in the small airways was 1038 (719-1500) $\mu \mathrm{g} / \mathrm{mL}$, which is 26 times larger than the threshold. Especially for TIP, the box plot shows that differences in deposition are relatively small.

In Figure 5, the concentrations of tobramycin in the extrathoracic and central airways are shown. Median (IQR) tobramycin concentration in the extrathoracic airways was $226,721(159,044-368,656) \mu \mathrm{g} / \mathrm{mL}$, which is 5668 times larger than the threshold value. Median concentration in the central airways was 44,378 $(23,142-65,819) \mu \mathrm{g} / \mathrm{mL}$, which is 1109 times larger than the threshold value.
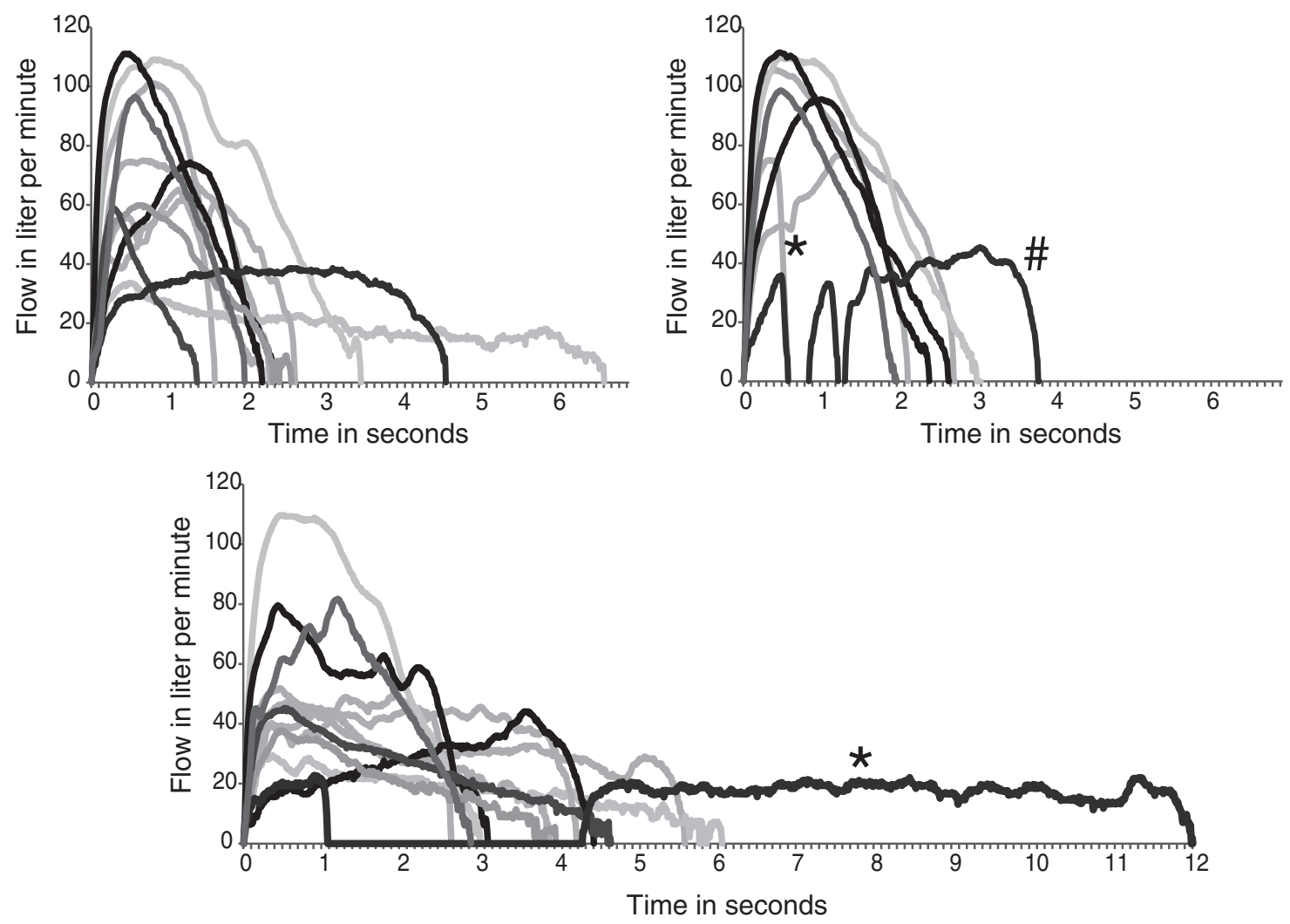

FIG. 2. Inspiratory flow curves of TIP. The inspiratory flow curves of TIP inhalations are shown for each patient. (a-c) Show the flow curves of the uninstructed, instructed fast, and instructed slow inhalations, respectively. The $x$-axis represents the time in seconds, and the $y$-axis represents the inspiratory flow in L/min. Each color represents a single patient. All study patients reached a higher PIF in the fast inhalation compared with the uninstructed inhalation, and lower PIFs in the slow inhalations compared with the uninstructed inhalations. (a) Inspiratory flow curves of uninstructed inhalations. (b) Inspiratory flow curves of instructed fast inhalations. *Patient coughed during inhalation. *Irregular inhalation. (c) Inspiratory flow curves of instructed slow inhalations. *Patient coughed during inhalation. PIF, peak inspiratory flow. 


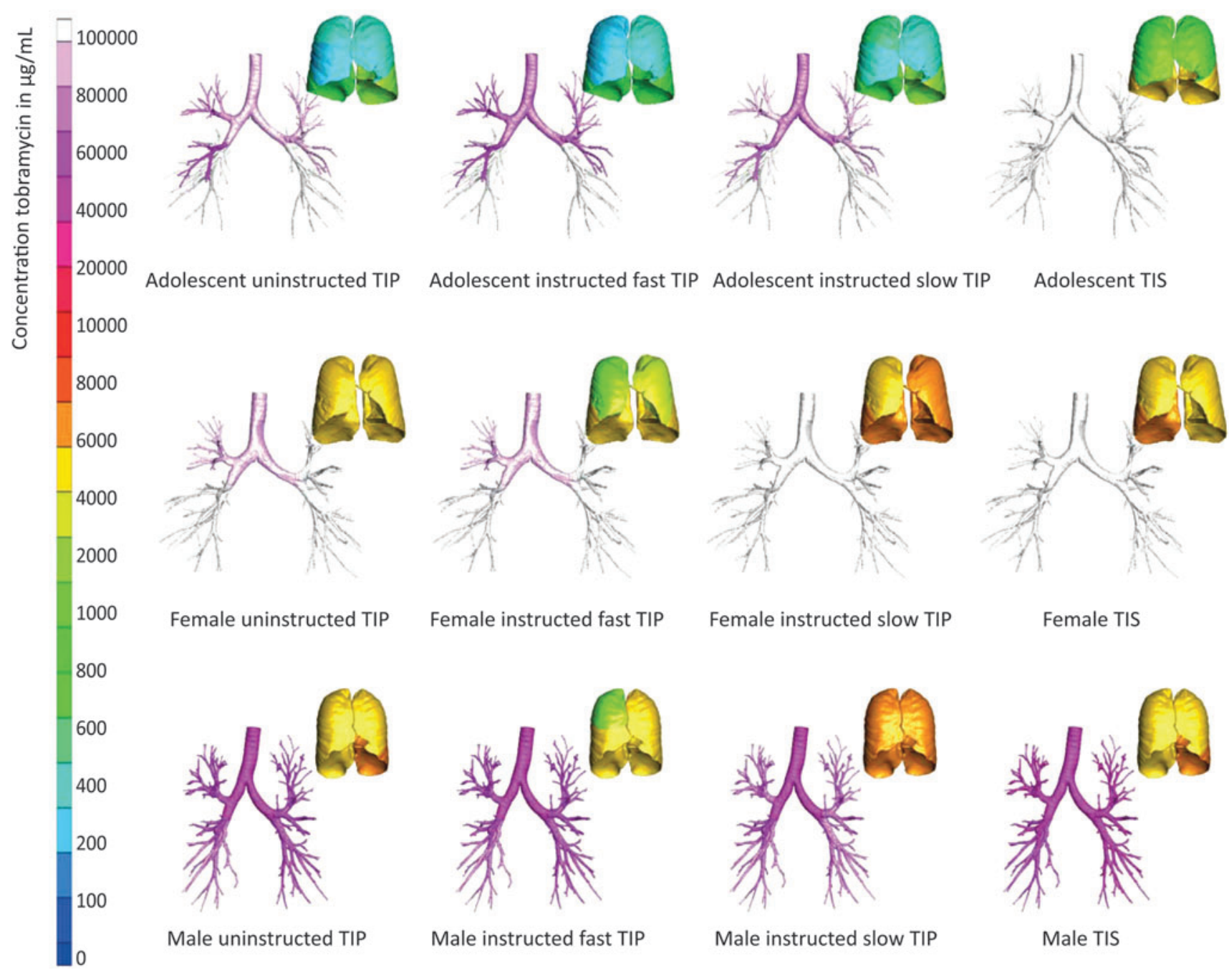

FIG. 3. Concentration of tobramycin in $\mu \mathrm{g} / \mathrm{mL}$ for all flow types in three single-study subjects. This figure shows 3D airway models constructed from CT scans of an adolescent (upper row), adult female (middle row), and adult male (lower row). The concentration is presented for each flow type: uninstructed, instructed fast, and instructed slow inhalation of TIP and the inhalations with TIS. The colors in the figures represent the tobramycin concentrations in $\mu \mathrm{g} / \mathrm{mL}$. The concentration in the central and large airways is shown in the image of the bronchial tree on the left, and the concentration in the small airways is indicated with the colored lung images in the right corners. White is the highest possible concentration, whereas dark blue represents the lowest concentration. Note the variability in deposition pattern between different flows within patients, and the very high concentrations in central and large airways. Furthermore, for these three patients, the minimal concentrations in the small airways were all above $200 \mu \mathrm{g} / \mathrm{mL}$, which is well above the threshold for effective concentrations of $40 \mu \mathrm{g} / \mathrm{mL}$.

Figures 4 and 5 show that compared with TIP, TIS particles were deposited deeper in the respiratory system, with less deposition in the extrathoracic region, and more deposition in the central, large, and small airways. The complete results of the generalized estimating equation models are presented in Supplementary Data S2. First, we assessed whether tobramycin concentrations differed between instructed inhalations of TIP or TIS inhalations and uninstructed TIP inhalations (Table a in Supplementary Data S2), with the latter being the reference in the generalized estimating equation model. Both instructed slow TIP inhalations and TIS inhalations resulted in significantly reduced extrathoracic airway concentrations $(p=0.024$ and $p<0.001)$, and greater central $(p=0.047$ and $p<0.001)$, large $(p=0.006$ and $p<0.001$ ), and small (both $p<0.001$ ) airway concentrations when compared with uninstructed TIP inhalations.
Instructed fast inhalations did not result in significantly different concentrations when compared with uninstructed inhalations for any airway region.

Second, we assessed whether tobramycin concentrations of all TIP inhalations different significantly from that of TIS inhalations (Table b in Supplementary Data S2), with TIS being the reference in the generalized estimating equation model. TIS inhalations resulted in significantly reduced concentrations in the extrathoracic airways and in significantly greater central, large, and small airway concentrations when compared with all TIP inhalations (all $p<0.001$ ), except for instructed slow TIP inhalations, which resulted in similar small airway concentrations as TIS.

When redoing the analysis excluding the two patients who did not reach a volume of $1.2 \mathrm{~L}$ in all their inhalations, 

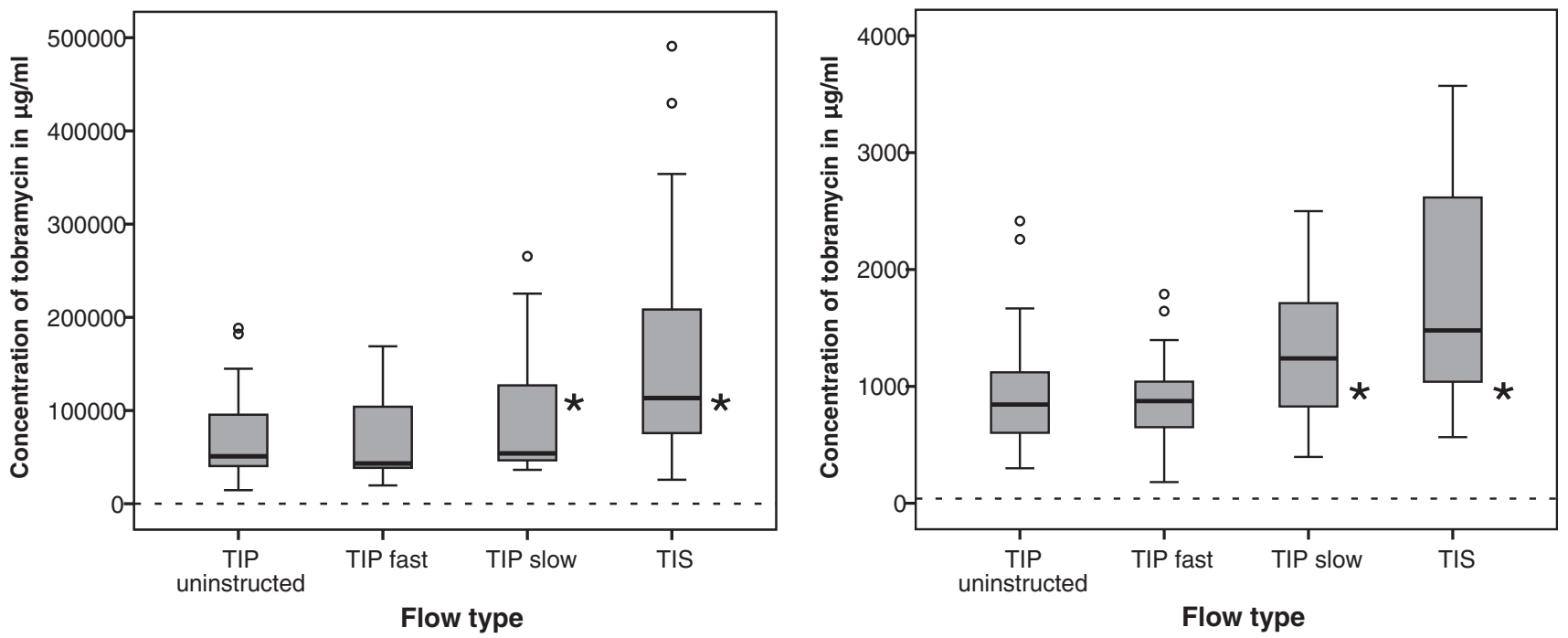

FIG. 4. Concentration of tobramycin in large and small airways, per flow type. Box-and-whisker plots of the concentration of tobramycin in $\mu \mathrm{g} / \mathrm{mL}$ in the large airways (a) and in the small airways (b). On the $x$-axis, the four different flow types are shown: uninstructed, instructed fast, and instructed slow inhalations with TIP and the inhalations with TIS. Note the difference in scale between the two graphs. The bolt line in each box represents the median; the bottom and top lines of the box represent the 25th and 75th percentile. The whiskers represent either 1.5 times the 25th or 75th percentile value or the minimum and maximum values. The circles outside of the T-bars are outliers. The dotted line in both graphs represents the threshold value for effective dose of 10 times MIC, that is, $40 \mu \mathrm{g} / \mathrm{mL}$. Concentrations that were significantly different from the concentrations of the uninstructed flow of TIP $(p<0.05)$ using generalized estimating equation models are marked with an asterisk. (a) Tobramycin concentration in the large airways. The instructed slow inhalations with TIP $(p=0.006)$ and the inhalations with TIS $(p<0.001)$ resulted in significantly greater concentrations of tobramycin, when compared with the uninstructed inhalation of TIP. (b) Tobramycin concentration in the small airways. The instructed slow inhalations with TIP and the inhalations with TIS resulted in significantly greater concentrations of tobramycin $(p<0.001$ in both cases), when compared with the uninstructed inhalation of TIP.

the outcomes of the generalized estimating equation models did not show different significant results.

\section{Effective tobramycin concentration}

In Supplementary Data S3, the deposition of tobramycin per lung lobe in the large and small airways is presented for both TIP and TIS. The tobramycin concentrations in both the large and small airways in all lung lobes were all well above the cutoff value for effective inhibition of $40 \mu \mathrm{g} / \mathrm{mL}$, assuming an airway surface liquid layer of $7 \mu \mathrm{m}$. For this airway surface liquid layer, of the patients who received a complete dose of TIP, the lowest local concentration of tobramycin in our simulations was found in the small airways in the left upper lobe of an adult female after an uninstructed inhalation of TIP. The tobramycin concentration was $128 \mu \mathrm{g} / \mathrm{mL}$, which is still more than triple the amount of the effective threshold concentration of $40 \mu \mathrm{g} / \mathrm{mL}$. The highest local concentration of tobramycin in the small airways was found in the left lower lobe of an adult male, after inhalation of TIS. The tobramycin concentration was $5656 \mu \mathrm{g} / \mathrm{mL}$, which is 141 -fold greater than the effective threshold concentration.

\section{Discussion}

We studied the effect of inhalation maneuvers and different formulations of tobramycin on the deposition throughout the lung with CFD, in a study population of CF patients of 12 years and older. Instructed slow inhalations of
TIP result in greater tobramycin concentrations in the large and small airways compared with inhalations of TIP without instruction. Furthermore, inhalations with TIS results in greater large and small airway concentrations when compared with all TIP inhalations, except for the instructed slow TIP inhalations. For the slow TIP inhalations, the small airway concentrations were similar to those of TIS. Importantly, all inhalations led to a concentration of tobramycin that was at least triple the amount of the effective clinical cutoff value of $40 \mu \mathrm{g} / \mathrm{mL}$. Furthermore, we conclude that it is feasible to instruct patients to perform specific inhalation maneuvers.

According to our results, the drug deposition of TIP is dependent on inspiratory flow rates. This finding contradicts the results of an earlier study that concluded that the T-326 inhaler is flow-rate independent, when experimentally measuring total lung doses at the level of the trachea with in vitro mouth throat deposition models. ${ }^{(17)}$ However, there were a few differences between the two studies. First, different throat models were used (no comparative studies between these models were performed). Furthermore, we assessed lung deposition for a larger number of flows: 36 compared with 10 flows that were selected from 152 flows of 38 patients in the earlier study. In addition, our flows showed a wider variation: we measured peak inspiratory flows of $30-113 \mathrm{~L} / \mathrm{min}$ compared with $49-88 \mathrm{~L} / \mathrm{min}$ in the study of Haynes et al. Hence, our results are likely to be representative of a more heterogeneous patient population. A flow-dependency investigation for the TIS formulation 


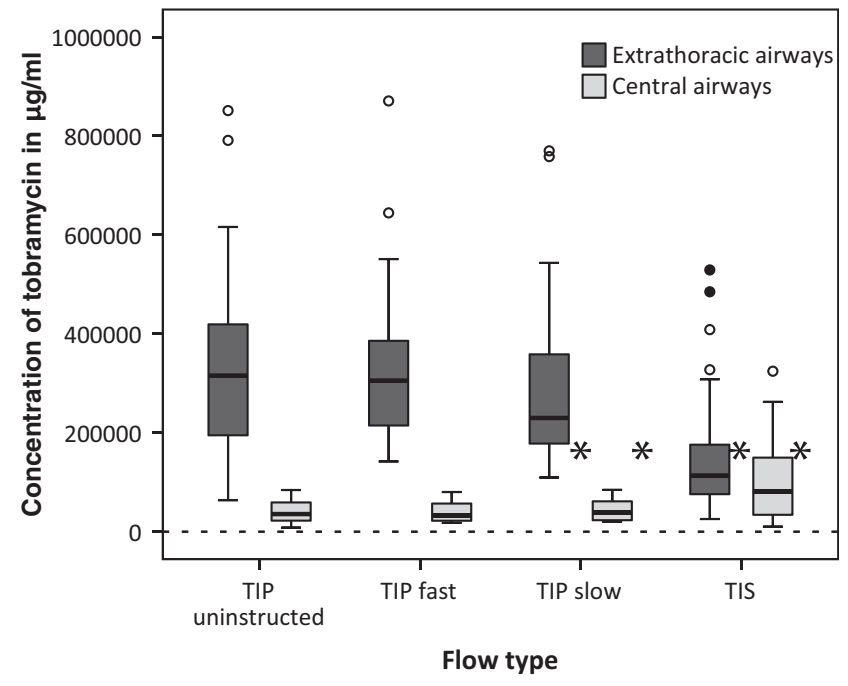

FIG. 5. Concentration of tobramycin in the extrathoracic and central airways, per flow type. Box-and-whisker plots of the concentration of tobramycin in $\mu \mathrm{g} / \mathrm{mL}$ in the extrathoracic and central airways (dark versus light box). On the $x$-axis, the four different flow types are shown: uninstructed, instructed fast, and instructed slow inhalations with TIP and the inhalations with TIS. The bolt line in each box represents the median; the bottom and top lines of the box represent the 25th and 75 th percentile. The whiskers represent either 1.5 times the 25 th or 75 th percentile value or the minimum and maximum values. The circles outside of the T-bars are outliers. The dotted line in both graphs represents the threshold value for effective dose of 10 times minimal inhibitory concentration, that is, $40 \mu \mathrm{g} / \mathrm{mL}$. Concentrations that were significantly different from the concentrations of the uninstructed flow of TIP $(p<0.05)$, using generalized estimating equation models, are marked with an asterisk. In the extrathoracic airways, the concentration of TIP was reduced after instructed slow TIP inhalations or inhalations with TIS $(p<0.001)$, compared with the uninstructed inhalation of TIP. Conversely, in the central airways, the instructed slow TIP $(p=0.047)$ and TIS $(p<0.001)$ inhalations resulted in greater tobramycin concentrations than the uninstructed inhalations of TIP.

was not part of the investigation, and so, the influence of individual flow patterns on the delivery of a nebulized solution remains unknown.

Results reported here show greater tobramycin deposition in large and small airways by TIS, when compared with TIP inhalations.

This finding contradicts results of a gamma scintigraphy study in healthy volunteers, more drug was delivered into the lungs with TIP than with TIS. ${ }^{(16,27,30)}$ There are several differences between the two studies. First, the gamma scintigraphy study was carried out with healthy volunteers, whereas the current study was carried out with CF patients. Second, the inspiratory flows for the TIS simulations were based on mean inhalation recordings of 15 seconds and assumed consistent, periodic inhalation cycles during the use of nebulizer for the complete treatment. When comparing the mass balances of tobramycin delivery with the PARI LC Plus nebulizer observed by Newhouse et al. ${ }^{(27)}$ with those from Lenney et al., ${ }^{(31)}$ the important relevance of the exhaled mass fraction on the much smaller lung deposition fraction can be detected. Even though device and extrathoracic losses are very similar in both publications, differences in patients or in their instruction can lead to different amounts of exhaled drug. As the computational modeling in this study did not account for exhalation once a drug particle reached the small airways, the amount deposited in these small airways and therefore the drug concentration might be overestimated for TIS. This modeling bias may explain the inconsistency with the results from scintigraphy studies and motivate further investigation on the sensitivity of nebulized therapies on variability of inhalation maneuver and its influence on deposited and exhaled fraction.

Uninstructed inhalations with TIP resulted in greater extrathoracic airway concentrations of tobramycin compared with TIS. This finding could be explained by the fact that the flows through the relatively small mouthpiece cross section of the T-326 inhaler are much higher than when inhaling quietly through the relatively wide PARI LC Plus nebulizer. This jet effect in combination with the higher inhalation rate results in greater velocity of the aerosol particles for TIP compared with TIS. The consequence is higher inertial forces and increased deposition of the inhaled aerosol particles in the oral cavity, which was already observed in the clinical deposition studies using scintigraphy. ${ }^{(27)}$ Greater extrathoracic deposition might also explain the observations reported by Konstan et al., who found greater sputum concentrations of tobramycin with use of TIP compared with TIS. ${ }^{(15)}$ Sputum concentrations are probably more representative for the extrathoracic deposition than for the deposition deeper in the respiratory system. High concentrations in the upper airways might be disadvantageous as increased numbers of fungal organisms (Candida albicans and Aspergillus species) are found in patients who nebulized TIS. ${ }^{(32)}$ Greater extrathoracic airway concentrations of tobramycin might further increase this effect.

On the contrary, greater concentrations in the lower airways might lead to increased serum concentrations of tobramycin, and overdosing of tobramycin can lead to nephrotoxicity and ototoxicity. However, in the pivotal studies of both TIP and TIS, it was described that trough and peak levels stay well below the potentially toxic levels. ${ }^{(10,33)}$ In our models, the differences in small airway concentrations are relatively small, and with these results it is highly unlikely that in patients in vivo the slow inhalations result in increased risk of toxicity relative to uninstructed inhalations. Uninstructed inhalations were also used in the pivotal studies.

However, in silico studies require many assumptions, which are potential confounders of our results. We will discuss the most important limitations of this study:

First, due to the fact that TIP is inhaled by single-breath inhalation and TIS is inhaled with continuous inhalations, different assumptions were made to perform the simulations for TIP and TIS. More specifically, for TIP we did not account for exhaled particles. TIS is inhaled with continuous breathing, and particles that were not deposited before exhalation phase were more likely to be exhaled. Furthermore, particles that were injected in the model during the exhalation phase were not inhaled at all. Besides, we did not account for differences in particle release between inspiratory and expiratory phases. Due to these different assumptions, the 
comparison between TIP and TIS might be less valid, since we do not know the effect of these assumptions.

Another limitation is the fact that CFD analyses are validated with SPECT-CT scan studies with asthma patients using nebulized aerosol particles at lower flow rates (30 L/min) that might behave differently than TIP PulmoSphere particles. ${ }^{(18)}$ Due to the porous formulation of TIP, the particles have a low contact surface area, reducing cohesive forces among particles, which prevents agglomeration. ${ }^{(16,17)}$ Therefore, our models might have led to an underestimation of the true deposition of TIP. We did not perform a SPECT-CT versus CFD validation study with $\mathrm{CF}$ patients using TIP PulmoSphere particles. That said, physical characteristics such as MMAD and its geometric standard deviation are the most decisive factors to predict aerosol behavior. By taking these characteristics of TIP into account, we fitted our simulations as closely as possible to the real situation.

Another limitation is the fact that the same throat model was used for all models instead of using the throats of patients. The geometry of the mouth and throat plays an important role in deposition of medication, and by standardizing the throat, this is not accounted for. Furthermore, we scaled the throat in such a way that it fitted our trachea, while there are more nuances in throat geometry between female and male adolescents, adult females, and adult males than the diameter at the trachea. However, the aim of our study was to assess the relationship between flow and lung deposition, and by limiting variation in other parameters, we could study this relationship with more precision.

We concluded that tobramycin concentrations are sufficiently high throughout the bronchial tree, using a clinical effective cutoff value of 10 times MIC. We decided to relate our results to the data provided by EUCAST (www.eucast .org/clinical_breakpoints/), that is, an MIC of tobramycin for $P a$ of $4 \mu \mathrm{g} / \mathrm{mL}$. Conservatively, we set our clinical cutoff value at 10 times $\mathrm{MIC}$ or $40 \mu \mathrm{g} / \mathrm{mL}$. Nevertheless, our clinical cutoff value at 10 times MIC does not necessarily mean that concentrations are high enough to be effective. Bos et al. described in a review that the effectiveness of inhaled antibiotics is influenced by multiple factors. The binding of thick $\mathrm{CF}$ mucus to tobramycin molecules actuates various mechanisms that can lead to a decreased number and/or inactivation of these molecules. ${ }^{(1)}$ Therefore, our in silico situation might be more positive that the in vivo situation.

Furthermore, we assumed that particles were distributed evenly over the small airway surface area. In reality this distribution is uneven, ${ }^{(34)}$ as some airways may be partially or totally occluded by mucus, resulting in no or less tobramycin deposition to the periphery of those airways, while other airways are open enabling inhaled particles to pass. Hence, our models of the small airways ignore the fact that some areas may not receive any tobramycin at all.

Patients are required to inhale TIP at least twice per capsule. However, of the 36 recorded inhalations with TIP, 34 were of a volume above the required $1.2 \mathrm{~L}$ to empty the content of a capsule (94\%). In our simulations, only one inhalation was simulated per occasion. Hence, we underestimated the delivered dose in the simulations because of the two patients who did not empty their capsule in one inhalation. However, when we performed a sensitivity analysis excluding these patients, the results were similar.

The 3D lung models were constructed with CT scans that were from other $\mathrm{CF}$ patients than the flow patients. In the Sophia Children's Hospital, CT scans are made of CF patients on routine basis biannually. This is not a national policy for $\mathrm{CF}$ health care in the Netherlands, and we did not have CT scans of all patients of other CF centers included in this study. Therefore, CT scans were selected from the database of Sophia Children's hospital, and matched for height.

Finally, in this study, the tobramycin deposition was assessed using CFD. We did not investigate whether the significantly higher small airway depositions after slow TIP inhalation or inhalations with TIS would result in better treatment effects.

In conclusion, in CF patients, 12 years and older, instructed slow inhalation of TIP resulted in greater concentrations of tobramycin in both the large and small airways of our models compared with uninstructed inhalation as well as instructed fast inhalations. When patients are instructed to inhale TIP slowly, the deposition in the small airways of our models is comparable with that of TIS. Importantly, all simulated inhalation maneuvers with both TIP and TIS resulted in concentrations well above a concentration of 10 times MIC for $P a$, even in the small airways. Careful and repeated instructions of patients regarding appropriate inhalation are important to target the small airways with an antibiotic deposition as high as possible.

\section{Acknowledgments}

We acknowledge the patients and their families who participated in the studies; C. Holsbeke and W. Vos of FLUIDDA NV for performing the CFS simulations; the local principal investigators of participating centers H.G.M. Arets, M.E. van der Eerden, C.J. Majoor, H.G.M. Heijerman; Novartis Pharma AG for technical input; and P. Robberts of Cresco Pharma for providing nebulizers. This study was supported by Novartis Pharma AG.

\section{Authors' Contributions}

J.J.M.: study protocol, study visits, and first article drafting; E.R.A.: statistical analyses; A.C.B.: study protocol; M.v.S., H.S., K.H., and P.M.: technical input in study protocol; H.A.W.M.T.: concept, study protocol, and principal investigator. All authors revised the article draft.

\section{Author Disclosure Statement}

J.J.M., E.R.A., and A.C.B.: no conflicts of interest exist; H.S.: employee of Novartis Pharma AG; M.v.S.: nonfinancial support from Siemens Healthineers, former consultant for Vertex Pharmaceuticals, Inc., outside the submitted work; K.H.: former employee of Novartis Pharmaceuticals Corporation; P.M.: employee of Novartis Pharma AG; H.A.W.M.T. reports grants from Roche, Novartis, CFF, Vertex, Gilead and Chiesi outside the submitted work, has a patent Vectura licensed, and a patent PRAGMA-CF scoring system issued and is heading the Erasmus MC-Sophia Children's Hospital core laboratory LungAnalysis. This work was supported by Novartis Pharma AG, Basel, Switzerland. 


\section{Funding Information}

This study was supported by Novartis Pharma AG.

\section{Supplementary Material}

Supplementary Data S1

Supplementary Data S2

Supplementary Data S3

\section{References}

1. Bos AC, Passe KM, Mouton JW, Janssens HM, and Tiddens HA: The fate of inhaled antibiotics after deposition in cystic fibrosis: How to get drug to the bug? J Cyst Fibros. 2017;16:13-23.

2. Foundation CF: Annual Data Report 2016, 2017. CFF.org/ research/researcher-resources/patient-registry/2016-patientregistry-annual-data-report.pdf. Last viewed on Feb. 4, 2020.

3. Society ECF: At-A-Glance Report 2016, Cystic Fibrosis in Europe-Facts and Figures 2016, 2018. ECFS.eu/sites/ default/files/general-content-files/working-groups/ecfs-patientregistry/CF1006_At_A_Glance_Guide_2016.pdf. Last viewed on Feb. 4, 2020.

4. Castellani C, Duff AJA, Bell SC, Heijerman HGM, Munck A, Ratjen F, Sermet-Gaudelus I, Southern KW, Barben J, Flume PA, Hodkova P, Kashirskaya N, Kirszenbaum MN, Madge S, Oxley H, Plant B, Schwarzenberg SJ, Smyth AR, Taccetti G, Wagner TOF, Wolfe SP, and Drevinek P: ECFS best practice guidelines: The 2018 revision. J Cyst Fibros. 2018;17:153-178.

5. Mogayzel PJ Jr., Naureckas ET, Robinson KA, Mueller G, Hadjiliadis D, Hoag JB, Lubsch L, Hazle L, Sabadosa K, Marshall B; and Pulmonary Clinical Practice Guidelines C: Cystic fibrosis pulmonary guidelines. Chronic medications for maintenance of lung health. Am J Respir Crit Care Med. 2013; 187:680-689.

6. Ratjen F, Munck A, Kho P, Angyalosi G, and Group ES: Treatment of early Pseudomonas aeruginosa infection in patients with cystic fibrosis: The ELITE trial. Thorax. 2010;65:286-291.

7. Ramsey BW, Pepe MS, Quan JM, Otto KL, Montgomery AB, Williams-Warren J, Vasiljev KM, Borowitz D, Bowman CM, Marshall BC, Marshall S, and Smith AL: Intermittent administration of inhaled tobramycin in patients with cystic fibrosis. Cystic Fibrosis Inhaled Tobramycin Study Group. N Engl J Med. 1999;340:23-30.

8. Smith WD, Bardin E, Cameron L, Edmondson CL, Farrant KV, Martin I, Murphy RA, Soren O, Turnbull AR, WierreGore N, Alton EW, Bundy JG, Bush A, Connett GJ, Faust SN, Filloux A, Freemont PS, Jones AL, Takats Z, Webb JS, Williams HD, and Davies JC: Current and future therapies for Pseudomonas aeruginosa infection in patients with cystic fibrosis. FEMS Microbiol Lett. 2017;364.

9. Geller DE, Konstan MW, Smith J, Noonberg SB, and Conrad C: Novel tobramycin inhalation powder in cystic fibrosis subjects: Pharmacokinetics and safety. Pediatr Pulmonol. 2007;42:307-313.

10. Konstan MW, Geller DE, Minic P, Brockhaus F, Zhang J, and Angyalosi G: Tobramycin inhalation powder for $P$. aeruginosa infection in cystic fibrosis: The EVOLVE trial. Pediatr Pulmonol. 2011;46:230-238.

11. Moore JE, and Mastoridis P: Clinical implications of Pseudomonas aeruginosa location in the lungs of patients with cystic fibrosis. J Clin Pharm Ther. 2017;42:259267.
12. de Jong PA, Nakano Y, Lequin MH, Mayo JR, Woods R, Pare PD, and Tiddens HA: Progressive damage on high resolution computed tomography despite stable lung function in cystic fibrosis. Eur Respir J. 2004;23:93-97.

13. Mott LS, Park J, Murray CP, Gangell CL, de Klerk NH, Robinson PJ, Robertson CF, Ranganathan SC, Sly PD, Stick SM, and Arest CF: Progression of early structural lung disease in young children with cystic fibrosis assessed using CT. Thorax. 2012;67:509-516.

14. De Backer JW, Vos WG, Devolder A, Verhulst SL, Germonpre P, Wuyts FL, Parizel PM, and De Backer W: Computational fluid dynamics can detect changes in airway resistance in asthmatics after acute bronchodilation. J Biomech. 2008;41:106-113.

15. Konstan MW, Flume PA, Kappler M, Chiron R, Higgins M, Brockhaus F, Zhang J, Angyalosi G, He E, and Geller DE: Safety, efficacy and convenience of tobramycin inhalation powder in cystic fibrosis patients: The EAGER trial. J Cyst Fibros. 2011;10:54-61.

16. Geller DE, Weers J, and Heuerding S: Development of an inhaled dry-powder formulation of tobramycin using PulmoSphere technology. J Aerosol Med Pulm Drug Deliv. 2011;24:175-182.

17. Haynes A, Geller D, Weers J, Ament B, Pavkov R, Malcolmson R, Debonnett L, Mastoridis P, Yadao A, and Heuerding $S$ : Inhalation of tobramycin using simulated cystic fibrosis patient profiles. Pediatr Pulmonol. 2016;51: 1159-1167.

18. De Backer JW, Vos WG, Vinchurkar SC, Claes R, Drollmann A, Wulfrank D, Parizel PM, Germonpre P, and De Backer W: Validation of computational fluid dynamics in CT-based airway models with SPECT/CT. Radiology. 2010;257:854-862.

19. Bos AC, van Holsbeke C, de Backer JW, van Westreenen M, Janssens HM, Vos WG, and Tiddens HA: Patientspecific modeling of regional antibiotic concentration levels in airways of patients with cystic fibrosis: Are we dosing high enough? PLoS One. 2015;10:e0118454.

20. Vinchurkar S, Backer LD, Vos W, Holsbeke CV, Backer JD, and Backer WD: A case series on lung deposition analysis of inhaled medication using functional imaging based computational fluid dynamics in asthmatic patients: Effect of upper airway morphology and comparison with in vivo data. Inhal Toxicol. 2012;24:81-88.

21. De Backer LA, Vos WG, Salgado R, De Backer JW, Devolder A, Verhulst SL, Claes R, Germonpre PR, and De Backer WA: Functional imaging using computer methods to compare the effect of salbutamol and ipratropium bromide in patient-specific airway models of COPD. Int J Chron Obstruct Pulmon Dis. 2011;6:637-646.

22. Vos W, De Backer J, Poli G, De Volder A, Ghys L, Van Holsbeke C, Vinchurkar S, De Backer L, and De Backer W: Novel functional imaging of changes in small airways of patients treated with extrafine beclomethasone/formoterol. Respiration. 2013;86:393-401.

23. Vos W, Backer Jd, Schroeder J, Sommerville M, Dwivedi SK, and Backer Wd: In-silico assessment of airway deposition using functional respiratory imaging for mono, dual and triple combination co-suspension metered dose inhaler formulations. C69. Here's looking at you: Imaging studies in copd. Am J Respir Crit Care Med. 2016;193:A5811.

24. Phalen RF, Oldham MJ, Kleinman MT, and Crocker TT: Tracheobronchial deposition predictions for infants, childern and adolescents. Ann Occup Hyg. 1988;32:11-21. 
25. Rosenow T, Oudraad MC, Murray CP, Turkovic L, Kuo W, de Bruijne M, Ranganathan SC, Tiddens HA, and Stick SM: PRAGMA-CF. A quantitative structural lung disease computed tomography outcome in young children with cystic fibrosis. Am J Respir Crit Care Med. 2015;191: 1158-1165.

26. Standaert TA, Speirs RJ, Rao N, Ung K, Tep V, Lin $\mathrm{T}$, Rourke AM, and Challoner P: Young cystic fibrosis patients can effectively use a novel high-payload capsule-based dry powder inhaler with tobramycin powder for inhalation (TPI). Pediatr Pulmonol. 2004;38: 284.

27. Newhouse MT, Hirst PH, Duddu SP, Walter YH, Tarara TE, Clark AR, and Weers JG: Inhalation of a dry powder tobramycin PulmoSphere formulation in healthy volunteers. Chest. 2003;124:360-366.

28. Tarran R, Button B, Picher M, Paradiso AM, Ribeiro CM, Lazarowski ER, Zhang L, Collins PL, Pickles RJ, Fredberg JJ, and Boucher RC: Normal and cystic fibrosis airway surface liquid homeostasis. The effects of phasic shear stress and viral infections. J Biol Chem. 2005;280:3575135759.

29. Tarran R, Button B, and Boucher RC: Regulation of normal and cystic fibrosis airway surface liquid volume by phasic shear stress. Annu Rev Physiol. 2006;68:543-561.

30. Challoner PB, Flora MG, Hirst PH, Klimowicz MA, Newman SP, Schaeffler BA, Speirs RJ, and Wallis SJ: Gamma scintigraphy lung deposition comparison of TOBI in the Pari LC Plus nebulizer and the Aerodose inhaler. Am J Respir Crit Care Med. 2001;163:A83.

31. Lenney W, Edenborough F, Kho P, and Kovarik JM: Lung deposition of inhaled tobramycin with eFlow rapid/LC Plus jet nebuliser in healthy and cystic fibrosis subjects. J Cyst Fibros. 2011;10:9-14.

32. Cheer SM, Waugh $\mathrm{J}$, and Noble S: Inhaled tobramycin (TOBI): A review of its use in the management of Pseudomonas aeruginosa infections in patients with cystic fibrosis. Drugs. 2003;63:2501-2520.

33. Geller DE, Pitlick WH, Nardella PA, Tracewell WG, and Ramsey BW: Pharmacokinetics and bioavailability of aerosolized tobramycin in cystic fibrosis. Chest. 2002;122: 219-226.

34. Kleinstreuer C, Zhang Z, and Donohue JF: Targeted drugaerosol delivery in the human respiratory system. Annu Rev Biomed Eng. 2008;10:195-220.

Received on February 15, 2019 in final form, November 6, 2019

Reviewed by: Ariel Berlinski Andy Clark

Address correspondence to: Harm A.W.M. Tiddens, MD, PhD Department of Pediatric Pulmonology and Allergology

Erasmus Medical Center Sophia Children's Hospital Postbus 2060, Room SP-3456

Rotterdam 3000 CB the Netherlands

E-mail: h.tiddens@erasmusmc.nl 